\title{
Sea Clutter Simulation Research based on Lognormal Distribution
}

\author{
Li Weibo ${ }^{a}$, Zhang Youzhi ${ }^{b}$, Zhang Guangyi ${ }^{c}$ \\ Naval Aeronautical Engineering Institute, Yantai, China, 264000 \\ awebblee2009@sina.com, byrerzyz@163.com, gyoei@qq.com
}

Keywords: Sea clutter; Stochastic process; Modeling and simulation; ZMNL

\begin{abstract}
The modeling and simulation of sea clutter is an important part of environmental simulation in radar target simulation. Sea clutter data got from simulation is good or not is the key to the optimal design of radar and radar signal processing. The presence of sea clutter will influence radar target detection and location tracking performance. Therefore, in the case of sea clutter as the main interference source, it is necessary to carry on the analysis of sea clutter characteristics in radar detection area. The paper gives some relevant characteristics of sea clutter, and sea clutter model and Zero Memory Nonlinearity (ZMNL) simulation method simulation under lognormal distribution.
\end{abstract}

\section{The concept and simulation method of sea clutter}

\section{The concept of sea clutter.}

The main function of radar system is target detection, namely discover a goal. On one or more radar coordinates, roughly locate the target. It is a method that radar can repeatedly measure target, track the target on its orbit, and can extrapolate to a future position. Estimate intercept point or drop point, and can extrapolate backward to estimate launch position.

But when radar detection is located in the goal on land or at sea, radar accepts not only object echoes, there are echoes from light area which are undesired. This part of the echo is called clutter in radar terms. Radar clutter is backscatter formed by radar beam shining on object's surface, and sea clutter is the clutter reflected back from the sea, which shows better dynamic characteristics. Sea surface, as reflector of radar wave, has very complex performances, and sea wind, sea current, wave, tide and different water quality and so on all have different influence on the production of sea clutter.

\section{The simulation method of sea clutter.}

When simulating clutter, we only need to produce a random number which has some characteristics of probability density and autocorrelation function, but this is not an easy thing to do. Simulation random sequence method with a certain probability distribution has been mature, but relevant random sequence method with certain probability density is under research. Now has Zero Memory Nonlinearity (ZMNL) is mature and reliable.

\section{The basic idea of ZMNL method}

ZMNL's basic idea is first to produce relevant Gaussian random process, and then after some nonlinear variable, get the desired random sequence.

Random sequence steps meeting the requirements of ZMNL method are as follows: first, Gaussian white noise sequence $\left\{\mathrm{V}_{\mathrm{i}}\right\},\left\{\mathrm{V}_{\mathrm{i}}\right\}$ through a linear digital filter $\mathrm{H}(\mathrm{z})$ and gets a sequence $\{\mathrm{Wi}\},\{\mathrm{Wi}\}$ after zero memory nonlinearity transformation $\mathrm{G}()$ and gets $\{\mathrm{Zi}\},\{\mathrm{Zi}\}$ 's distribution characteristics can be got by nonlinear transformation $\mathrm{G}($ ), and digital filter $\mathrm{H}(\mathrm{z})$ is used to satisfy spectrum characteristics.

Input Gaussian white noise sequence $\left\{\mathrm{V}_{\mathrm{i}}\right\}$, through linear system $\mathrm{H}(\mathrm{z})$ still subjects to Gaussian distribution, and power spectrum function is the square of system amplitude frequency function. FG () is for the distribution function value of every random value input to Gaussian distribution sequence by Gaussian distribution function. Its output sequence will be $(0,1)$ uniform distribution sequence. Then by means of nonlinear transformation F - 1 (), get the sequence meeting the 
requirements. F - 1 () is the inverse function of probability distribution function of amplitude fluctuation model, and it guarantees the distribution characteristics of output random sequence.

Therefore, the general steps of ZMNL method can be expressed as follows:

1) Produce Gaussian white noise sequence $\left\{V_{i}\right\}$;

2) Gaussian white noise sequence $\left\{\mathrm{V}_{i}\right\}$ goes through a linear filter $H(z)$, and gets Gaussian noise sequence $\{\mathrm{Wi}\}$, which is to make random variables $\left\{\mathrm{V}_{\mathrm{i}}\right\}$ of all time points have certain correlations;

3) For relevant Gaussian noise sequence $\{\mathrm{Wi}\}$, nonlinear transform gets some probability distribution related sequence $\{\mathrm{Zi}\}$.

The principle block diagram can be expressed as follows:

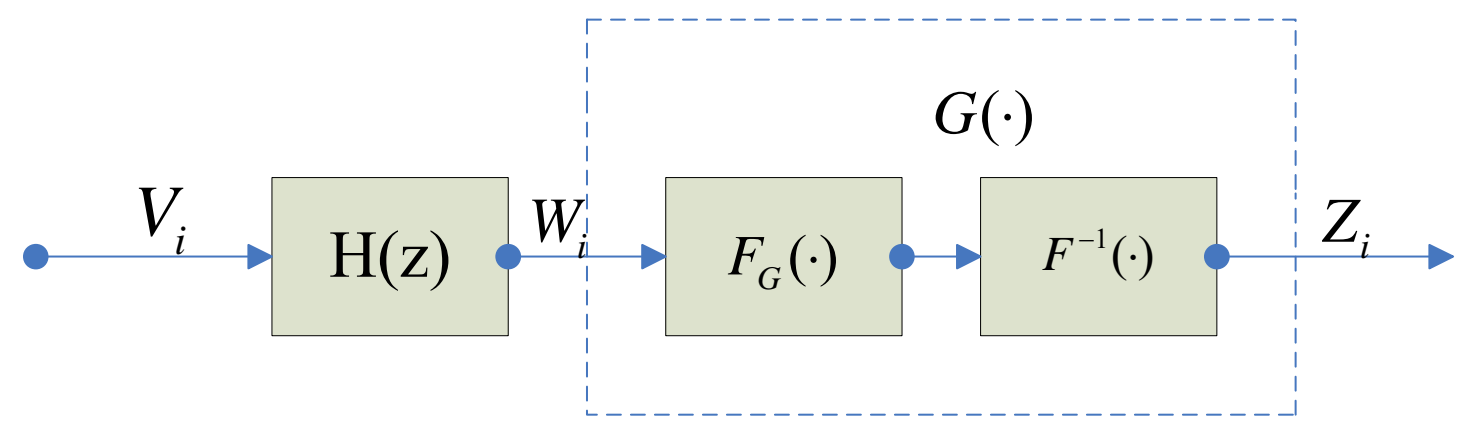

Figure 1 ZMNL method principle block diagram

If ZMNL is a polynomial representation, ZMNL's input is limited, and output is limited. For ZMNL which cannot be expressed by Polynomial representation, input is Gaussian random process, and any ZMNL can smoothen and extend its output spectrum. Therefore, by studying the relationship between $Z M N L$ input $\{\mathrm{Wi}\}$ and output $\{\mathrm{Zi}\}$ 's correlation functions $\rho_{i j}$ and $S_{i j}$ :

$$
S_{i j}=\frac{E\left[Z_{i} Z_{j}\right]-E\left[Z_{i}\right] E\left[Z_{j}\right]}{\sqrt{D^{2}\left[Z_{i}\right] D^{2}\left[Z_{j}\right]}}
$$

In it, $\mathrm{i}, \mathrm{j}=1,2,3, \ldots, \mathrm{N}$.

$$
\rho_{i j}=\frac{E\left[W_{i} W_{j}\right]-E\left[W_{i}\right] E\left[W_{j}\right]}{\sqrt{D^{2}\left[W_{i}\right] D^{2}\left[W_{j}\right]}}
$$

In it, $\mathrm{i}, \mathrm{j}=1,2,3, \ldots, \mathrm{N}$.

Use $\{\mathrm{Zi}\}$ 's correlation function $S_{i j}$ to calculate the correlation function $\rho_{i j}$ of $\{\mathrm{Wi}\}$, by $S_{\omega}(\omega)=F\left\{E\left(\omega \omega_{j}\right)\right\} c_{2}$ (F is Fourier transform), $H(\omega)$ is obtained.

The beauty of this approach is that Gaussian white noise's distribution is Gaussian after going through linear filter, and operation is of high efficiency with stable performance. The difficulty is the design of $\mathrm{H}(\mathrm{z})$, because of nonlinear transformation can make input sequence $\{\mathrm{Wi}\}$ spectral broadening, and make the autocorrelation functions of input sequence $\{\mathrm{Wi}\}$ and output sequence $\{\mathrm{Zi}\}$ has a very complex transformation relationship. So we must find out the nonlinear correlation relationships of input sequence and output sequence, and their corresponding correlation determination is related with distribution form, and function simulation is implemented. ZMNL transformation cannot provide independent control of probability density function and correlation function, and make both difficult to adjust at the same time.

This study introduces clutter modeling which is built on the assumption of clutter is a generalized stationary random process. Simplify clutter simulation to a stochastic process which at the same time has particular probability density (PDF) and power spectral density (PSD). So the socalled clutter simulation is to find a fast algorithm to produce both to certain random sequence satisfying certain range and related properties. Simulation method of random sequence with certain 
probability distribution is mature, but methods which can produce relevant random sequence with certain probability density currently is ZMNL and SIRP, and give simulation results of ZMNL.

\section{The generation of lognormal distribution clutter's ZMNL}

Sea clutter is uniform, and for radar performance, it is a statistical phenomenon. For low resolution radar (antenna beam width is greater than 2 and pulse width is greater than $1 \mu$ ), sea clutter amplitude generally obeys Rayleigh amplitude distribution. In high resolution radar, it is often observed that magnitude generally obeys logarithmic normal distribution and Weibull distribution and related $\mathrm{K}$ distribution. Specific implementation method of lognormal distribution clutter's ZMNL is given here:

When radar's flair is improving or under high sea condition, the tail of the cluster is longer, and backscatter features are off Rayleigh distribution, which is more in line with amplitude distribution of logarithmic normal distribution, probability density function is:

$$
f_{d}(x)=2\left(2 \pi \sigma_{c}^{2} x^{2}\right)^{-\frac{1}{2}} \exp \left[\frac{-2\left(\ln \frac{x}{x_{m}}\right)^{2}}{\sigma_{c}^{2}}\right], x>0, x_{m}>0, \sigma_{c}>0
$$

In it, $x_{m}$ is scale parameter, which is the median; $\sigma_{c}$ is shape parameter, which is the standard deviation of $\ln x^{2}$, which is the distribution. When radar identification resolution

improves or is under high sea condition, the backward scattering properties of sea clutter are off Rayleigh distribution. Its tail is long, which is available to logarithmic normal distribution fitting. Here estimate according to measured data $\sigma_{c}$.

From the above equations, define that relation between $S_{i j}$ and $\rho_{i j}$ is as follows:

$$
\rho_{i j}=\frac{\ln \left[1+S_{i j}\left(e^{\sigma_{c}^{2}}-1\right)\right]}{\sigma_{c}^{2}}
$$

$\mathrm{H}$ (omega) can be calculated.

According to the introduction, ZMNL clutter modeling and simulation process steps can be summarized as follows:

(1) Give shape parameters and spectrum for the clutter distribution simulation;

(2) Calculate the autocorrelation function of Gaussian colored noise by nonlinear transformation relationship in front and back of the autocorrelation function.

(3) Get the structure of linear filter according to the autocorrelation function of Gaussian colored noise with modern spectrum estimation method;

(4) Get Gaussian white noise and then get clutter according to the process of ZMNL method.

In this section, with comprehensive probability distribution and power spectrum knowledge, perform sea clutter simulation according to the above steps, simulation results are given and analyzed.

\section{Simulation of logarithmic normal distribution}

For normal distribution, get $\sigma_{f}=40$, and probability density shape parameters $\sigma_{c}=0.6 \mathrm{db}$, and the simulation results are as follows: 


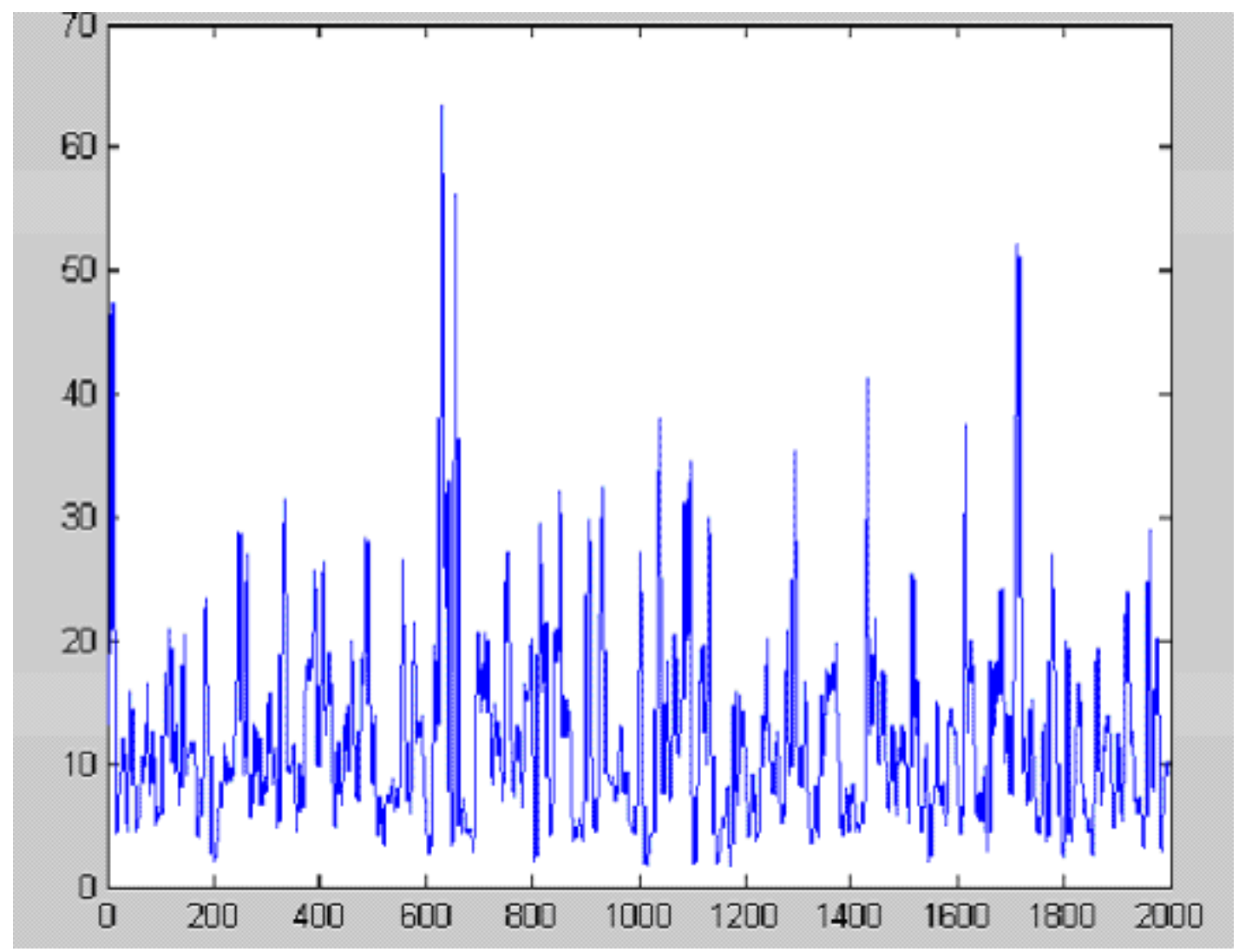

Figure 2 Lognormal distribution clutter domain waveform

\section{References}

[1] Oliver CJ.Correlated K-Distributed clutter models.Optical Acta,2005,32(12):1515 1547 2001,30(12):1 5

[2] Kay SM.Modern spectrum estimation:Theory and application.Prentice Hall,1988:543

[3] R.L. Mitchell, Chen Xunda translation. Radar system simulation. Beijing: Science Press, 1982

[4] Ward KD.Radar sea clutter.Microwave Journal,2002(6):25 32

[5] Rangaswamy M,Weiner D.Computer generation of correlated Non-Gaussian radar clutter. IEEE Transactions on Aerospace and Electron System, 2005.1 